EXTENDED REPORT

\title{
Influence of change in body position on choroidal blood flow in normal subjects
}

\author{
P Kaeser, S Orgül, C Zawinka, G Reinhard, J Flammer
}

Br J Ophthalmol 2005;89:1302-1305. doi: 10.1136/bjo.2005.067884

See end of article for authors' affiliations

.....................

Correspondence to:

Dr S Orgül, University Eye

Clinic Basel, Mittlere

Strasse 91, $\mathrm{P} O \mathrm{O}$ Box, $\mathrm{CH}$

4012 Basel, Switzerland;

sorguel@uhbs.ch

Accepted for publication 1 April 2005

\begin{abstract}
Aim: To compare subfoveal choroidal blood flow (ChBF) in sitting and supine positions in normal
\end{abstract} volunteers.

Methods: ChBF was measured with laser Doppler flowmetry in 22 healthy volunteers of mean (SD) age 24 (5) years. Six independent measurements of ChBF were obtained in one randomly selected eye of each subject while seated. The subjects then assumed a supine position for 30 minutes and a new series of six measurements was obtained. The mean values of the two series were calculated. Systemic brachial artery blood pressure and intraocular pressure were measured in the sitting and supine positions. Ocular perfusion pressure (OPP) was calculated based on formulae derived from ophthalmodynamometric studies. The influence of changing OPP during change in body posture on the change in ChBF was assessed by linear regression analysis.

Results: ChBF decreased by $6.6 \%(p=0.0017)$ in the supine position. The estimated ophthalmic blood pressure in the supine position was adjusted to obtain a result of no change in OPP for no change in ChBF, yielding a mean decrease in the estimate of OPP of $6.7 \%(p=0.0002)$. The necessary adjustment for the estimate of OPP in the supine position suggested a marked buffering of the change in perfusion pressure by the carotid system. The relative decrease in OPP correlated significantly with the relative decrease in $\operatorname{ChBF}\left(R^{2}=0.20 ; p=0.036\right)$ with a slope for the regression line of 1.04 .

Conclusions: The comparable degree of change in ChBF and OPP and the linear relationship between the two parameters suggest a passive response of the choroidal circulation to a change in posture. In contrast, the carotid system seems to control the gradient in perfusion pressure closely between the heart and its branches.
T he functional integrity of the retina depends entirely on an adequate vascular perfusion level and an availability of vital metabolic nutrients and oxygen. In humans these factors are promoted by a dual vascular system. The avascular region of the retinal fovea, however, relies entirely on blood flow in the subfoveal choroidal vasculature and, more specifically, in the choriocapillaris. The understanding of choroidal blood flow (ChBF) regulation is therefore of utmost importance when considering the pathophysiology of diseases such as age related macular degeneration, diabetic retinopathy, and others.

Notwithstanding the concept of a passive vascular system, investigations in rabbits have shown a non-linear relationship between $\mathrm{ChBF}$ and perfusion pressure. ${ }^{1}$ Even some studies in humans have suggested that ChBF may be partly independent of perfusion pressure. ${ }^{23}$ On the other hand, a recent study suggested a passive response of the choroidal circulation to perfusion pressure changes during changes in body position. ${ }^{4}$ Because the alteration of body posture was only maintained for a few minutes in this latter study, it is possible that some control mechanisms may not have had time to take effect. We therefore investigated the response of the subfoveal ChBF in healthy subjects in the sitting position and 30 minutes after assuming a supine position.

\section{METHODS}

\section{Compact choroidal laser Doppler flowmeter}

ChBF assessment was performed using laser Doppler flowmetry. ${ }^{5}$ A confocal choroidal laser Doppler flowmeter (Choroidal Blood Flowmeter; IRO, Sion, Switzerland) was used. The optical system for the delivery of the laser beam and the detection of the scattered light is based on a confocal arrangement and has been described in detail elsewhere. ${ }^{6}$
With this instrument, the scattered light is collected from within the avascular zone of the fovea and ChBF in the subfoveal region is computed. ${ }^{2-10}$

\section{Subjects}

Twenty two healthy non-smoking subjects (19 women) of mean (SD) age 24.1 (4.7) years (range 18-40) were recruited from volunteers in the University Eye Clinic, Basel. Based on previous studies, ${ }^{411}$ this sample size was considered to provide sufficient statistical power in the present study. The procedures were approved by the local ethical committee, the tenets of Declaration of Helsinki were followed, and each subject signed an informed consent form before any examination.

Subjects were screened for ocular and systemic diseases. A detailed medical and ophthalmic history was recorded, and all subjects completed an ophthalmologic examination. Only individuals with no history of ocular or systemic disease, no history of chronic or current systemic or topical medication, and no history of drug or alcohol abuse were included in the study. Further inclusion criteria were a normal systolic blood pressure (SBP, 100-140 mm Hg) and diastolic blood pressure (DBP, 60-90 mm Hg), best corrected visual acuity above 20/ 25 in both eyes, ametropia within -3 to +3 dioptres of spherical equivalent and less than 1 dioptre astigmatism in both eyes, a pupil diameter of at least $4 \mathrm{~mm}$, an applanatory (Goldmann applanation tonometry) intraocular pressure

Abbreviations: $\mathrm{ChBF}$, choroidal blood flow; DBP, diastolic blood pressure; IOP, intraocular pressure; MBP, mean arterial blood pressure; OAP, ophthalmic arterial blood pressure; OPP, ocular perfusion pressure; SBP, systolic blood pressure 
(IOP) below $20 \mathrm{~mm} \mathrm{Hg}$ in both eyes, and no pathological findings on slit lamp examination and indirect fundoscopy.

\section{Experimental procedure}

Subjects were studied at least 2 hours after a light meal and were asked to refrain from alcohol and caffeine for 12 hours before the trial days, as well as to avoid heavy exercising or meals too rich in sugar and fat before the experimental day. A resting period of at least 30 minutes was scheduled for each subject. Stable baseline conditions were established, which were ensured by repeated measurements of blood pressure (Profilomat, Roche, Basel, Switzerland). After stabilisation of blood pressure, two measurements of SBP and DBP as well as heart rate (HR) were recorded and averaged. Immediately afterwards, IOP was determined with a handheld tonometer (Tonopen; Mentor, Norwell, MA, USA) after applying one drop of $0.4 \%$ benoxinate hydrochloride and staining the tear film with a strip of fluorescein sodium. The subjects were seated with the head stabilised in a slit lamp head rest. Care was taken to standardise the subject's head position which was held constant throughout the recordings by aligning marks on the head rest with anatomical landmarks on the head. The subjects were asked to fixate the red light spot within the ocular and to adjust the focus by turning the ocular. The ocular-to-cornea distance was set between 1.5 and $2 \mathrm{~cm}$ and held constant throughout the recordings. In addition, a constant very low level artificial room illumination was used throughout all the experiments. The experimental eye was chosen randomly and measurements were obtained without pupil dilatation. A stable direct current during a recording was used as a criterion of proper fixation. ${ }^{12}$

The same investigator performed all ChBF measurements (PK). A first series of six independent consecutive recordings of 20-25 seconds each was obtained in a sitting position, with the subjects taking their head off the chin rest and the examiner moving the laser Doppler flowmeter between the measurements. ${ }^{13}$ Individual measurements were obtained at least 1 minute apart. The subjects then assumed a supine position for at least 30 minutes and, after stabilisation of blood pressure, IOP, SBP, DBP, and HR were determined as outlined above. The laser Doppler flowmeter mounted on a swivel arm was rotated by $90^{\circ}$ and the camera was aligned to the subject's eye, based on the landmarks identified in the sitting position. A second series of six independent measurements was obtained in the supine position and the mean ChBF value was calculated for both series.

\section{Statistics}

The SBP and DBP readings were used to calculate the mean arterial blood pressure (MBP) according to the formula: MBP $=\mathrm{DBP}+0.42(\mathrm{SBP}-\mathrm{DBP}) .{ }^{4}{ }^{14}$ Mean ophthalmic arterial blood pressure (OAP) was calculated based on formulae derived from ophthalmodynamometric studies, with mean $\mathrm{OAP}$ in the sitting position calculated as $\mathrm{OAP}=0.74 \times \mathrm{MBP}$ and OAP in the supine position calculated as OAP $=0.84 \times$ MBP. ${ }^{414} 15$ The mean ocular perfusion pressure (OPP) was calculated according to the formula: OPP $=$ OAP - IOP. The differences between sitting and supine positions were calculated for OPP and ChBF by accounting for heteroscedasticity. ${ }^{16}$ Departure from normal distribution of the variables was assessed by means of the Shapiro-Wilk W test for normality and measurements in the sitting position were compared with those in the supine position using a paired $t$ test. The dependence of change in ChBF on change in OPP was assessed by computing the significance of the slope of the line regressing the percentage difference in ChBF on the percentage difference in OPP. A $p$ value of $\leqslant 0.05$ was considered statistically significant.

\section{RESULTS}

The distribution of the data for ChBF in the sitting $(p=0.28)$ and supine positions $(p=0.46), M B P$ in the sitting $(p=0.91)$ and supine positions $(p=0.43)$, HR in the sitting $(p=0.69)$ and supine positions $(p=0.49)$, and OPP in the sitting $(p=0.63)$ and supine positions $(p=0.28)$ was normal. Changes in $\mathrm{ChBF}, \mathrm{MBP}, \mathrm{HR}$, and OPP were also normally distributed $(p=0.86, p=0.09, p=0.13$, and $p=0.78$, respectively). The variation (coefficient of variation) between the two series of ChBF measurements was comparable $(10.28 \%$ and $9.58 \%$ in the sitting and supine positions, respectively: $\mathrm{p}=0.58$, paired $t$ test). The mean (SD) results for the measured parameters IOP, SBP, DBP, MBP, HR, and ChBF are shown in table 1. MBP decreased by $7.8(4.9) \%$, HR by 8.2 (9.6)\%, and ChBF by 6.6 (11.6)\%. The mean (SD) estimated OPP was 50.7 (5.9) $\mathrm{mm} \mathrm{Hg}$ and 53.2 (4.8) $\mathrm{mm} \mathrm{Hg}$ in the

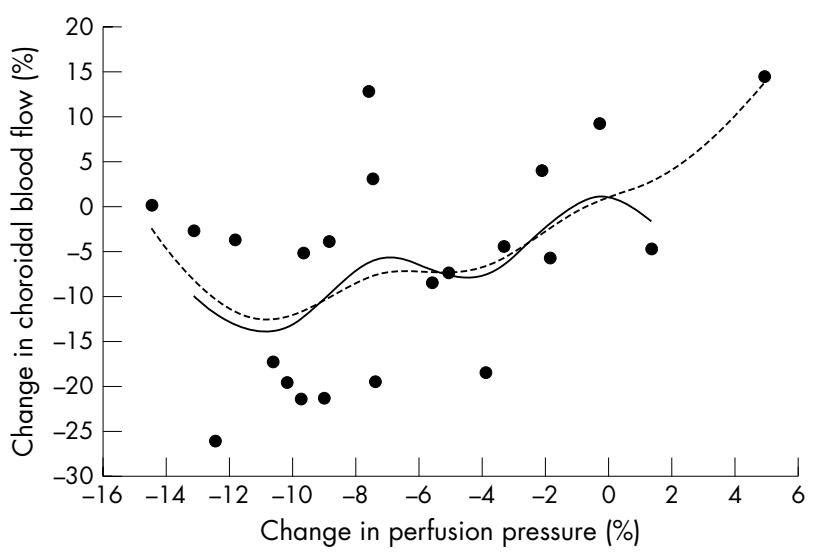

Figure 1 A curve fitted according to the distance-weighted least squares smoothing procedure and regressing the relative change in choroidal blood flow on the relative change in ocular perfusion pressure (dotted line) suggested a nearly linear fit except for the lowest end of the curve, suggesting an outlier. When the procedure was repeated after excluding the extreme points at the upper and lower end (solid line), the curve showed a steady decrease in choroidal blood flow with decreasing ocular perfusion pressure.

Table 1 Mean (SD) parameters in the sitting and supine positions

\begin{tabular}{llll}
\hline & Sitting position & Supine position & p value* \\
\hline IOP $(\mathrm{mm} \mathrm{Hg})$ & $13.0(2.2)$ & $14.0(2.1)$ & 0.0006 \\
SBP $(\mathrm{mm} \mathrm{Hg})$ & $106.2(12.6)$ & $102.7(7.2)$ & 0.046 \\
DBP $(\mathrm{mm} \mathrm{Hg})$ & $71.5(8.6)$ & $63.6(6.9)$ & 0.0001 \\
MBP $(\mathrm{mm} \mathrm{Hg})$ & $86.1(8.7)$ & $80.0(6.2)$ & 00.0001 \\
Heart rate (beats/min) & $64.1(8.1)$ & $59.0(10.1)$ & 0.0006 \\
ChBF (arbitrary units) & $9.5(2.2)$ & $8.9(2.1)$ & 0.0017 \\
\hline
\end{tabular}

ChBF, choroidal blood flow; DBP, diastolic blood pressure; IOP, intraocular pressure; MBP, mean arterial blood pressure; SBP, systolic blood pressure.

*Paired $t$ test. 


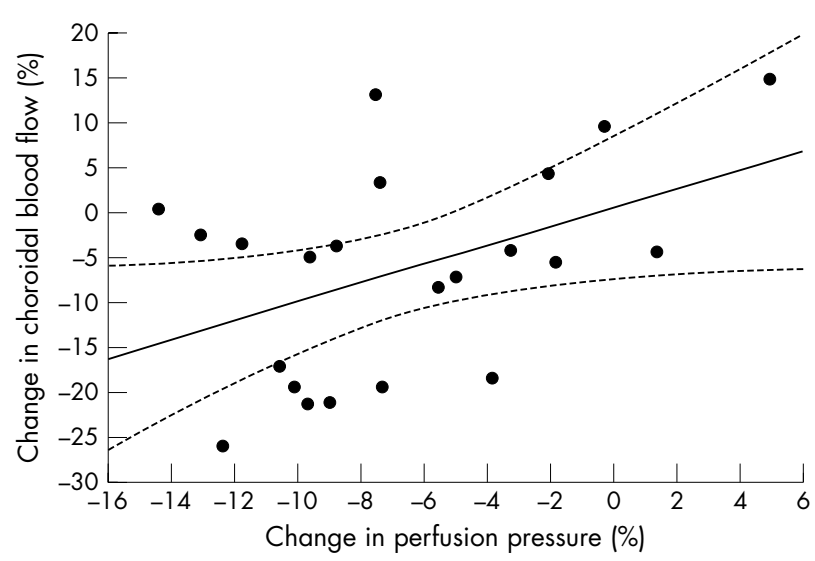

Figure 2 Relative change in ocular perfusion pressure correlated linearly with the relative change in choroidal blood flow $\left(R^{2}=0.20\right.$, $p=0.036$ ).

sitting and supine positions respectively $(\mathrm{p}=0.0005)$, with a mean (SD) increase of $5.4(5.6) \%$.

Because of a negative mean change in ChBF during an estimated mean increase in OPP, it was hypothesised that mean OAP in the supine position had been overestimated. Considering the measured parameters in table $\mathrm{l}$ and an adjusting factor for the estimate of the mean OAP in the sitting position of 0.74 , a new adjusting factor for the estimate of the mean OAP in the supine position was calculated in order to obtain no change in perfusion pressure for no change in ChBF. Solving the equation yielded an adjusting factor of 0.767 instead of 0.84 . With the new adjusting factor for the estimate of the mean OAP in the supine position, the mean (SD) OPP was 50.7 (5.9) $\mathrm{mm} \mathrm{Hg}$ and 47.3 (4.4) $\mathrm{mm} \mathrm{Hg}$ (the latter parameter was still normally distributed: $\mathrm{p}=0.31$ ) in the sitting and supine positions respectively $(p=0.0002)$, with a mean (SD) decrease of 6.7 (5.0)\%.

Scrutinising distance-weighted least squares curves regressing change in ChBF on change in OPP (fig 1), a steady decrease in ChBF with decreasing OPP was observed. A linear fit was therefore computed to the entire data set and the relative change in OPP correlated positively with the relative change in ChBF $\left(R^{2}=0.20, p=0.036\right.$; fig 2$)$. Residual analysis applying the $2 \times$ sigma limit did not unveil outliers in this regression analysis which showed a slope of 1.04.

\section{DISCUSSION}

This study assessed the response of subfoveal ChBF to change in body posture. The experimental set up was designed to provoke a change in OPP while limiting neurovegetative input during change in perfusion pressure. Enough time was scheduled after the subjects had assumed the supine position to allow the regulatory mechanisms to take effect. The change from a sitting position to a supine position resulted in a change in ChBF which depended linearly on the change in OPP, indicating a passive response of the choroidal circulation to changes in OPP. This finding suggests that the choroidal vasculature has no intrinsic ability to regulate blood flow during changes in OPP. However, the experimental data suggest that the carotid system is able to buffer the increase in perfusion pressure in the ophthalmic artery.

A number of regulatory systems and factors such as circulating hormones, as well as metabolic, myogenic and neurogenic factors, participate in the regulation of vascular tone.$^{17}$ Autoregulation is said to occur when, within certain limits, the effect of the decrease in perfusion pressure is compensated by a decrease in vascular resistance and the flow remains constant. A number of studies have shown that the human retina has some autoregulatory capacity in response to both an increase and a decrease in OPP. In contrast to the choroid, the retina does not have neurovegetative innervation so the elicited vascular response during change in perfusion pressure will be modulated by mechanisms intrinsic to the vessels, whereas the choroidal vasculature may experience an important neuronal influence. Investigating blood flow autoregulation in the choroid may therefore be challenging. While earlier studies had suggested a linear pressure-flow relationship in the choroid of primates, ${ }^{18} 19$ more recent studies have implied some autoregulatory capacity in the choroid of rabbits. ${ }^{1}$ Even in humans, the response of the ChBF observed after changing the OPP with a suction cup suggested some autoregulatory capacity in the choroidal vasculature. ${ }^{2}$ A non-linear relationship between the genuine OPP and ChBF in a normal population seemed to concur with such an interpretation. ${ }^{3}$ Finally, taking advantage of the increase in systemic blood pressure resulting from neurovegetative stimulation during isometric exercise, several studies have shown relative stability of the ChBF during increased OPP. ${ }^{8}{ }^{10}$ However, it must be remembered that the vascular response in the choroid after exercise induced changes in OPP may be driven by direct sympathetic input to the choroid and not represent an autoregulatory response intrinsic to the choroidal vessels. Furthermore, a recent study-which also avoided direct interventions on the eye such as applying a suction cup or stimulation of the neurovegetative system-found a passive response of the choroidal vasculature to changes in perfusion pressure. ${ }^{4}$ The inconsistency between the latter finding and an earlier result from the same group ${ }^{2}$ suggests that the use of means such as a suction cup to increase IOP may elicit mechanisms responsible for a non-linear pressure-flow relationship in the choroid.

In the present study a relative increase in OPP was observed when estimates of the blood pressure in the ophthalmic artery were calculated with the same factors as in a previous study. ${ }^{4}$ Longo et al found that the increase in calculated OPP estimated using data based on ophthalmodynamometric measurements was much less than the increase calculated with the hydrostatic model and, because the estimated change in perfusion pressure fitted the measured change in ChBF, they hypothesised an active mechanism acting between the heart and the eye, buffering the increase in OPP. It is likely that this effect-possibly based on myogenic mechanisms which usually need more time to take effect ${ }^{20}{ }^{21}$ - may have been even more marked when the subjects were allowed to rest for 30 minutes in the supine position. Obviously, the estimate of change in OPP was erroneous and with a correcting factor of 0.767 instead of 0.84 in the supine position, a zero change in OPP corresponded to a zero change in ChBF. This suggests some external control mechanisms regulating the OPP in the ophthalmic artery, similar to the control mechanisms in the carotid system. ${ }^{22}$ Consequently, the change in the gradient in OPP between the heart and the cerebral circulation is buffered in the supine position. As expected, ${ }^{14}$ a reduction in systemic blood pressure was observed after assuming the supine position and OPP dropped. The decrease in OPP and ChBF were of similar amplitude and amounted to $-6.7 \%$ and $-6.6 \%$ respectively, confirming the findings of Longo et al. Furthermore, the regression line between the two variables showed a slope of 1.04 indicating an absence of a regulatory response within the choroid.

We confirmed the findings of Longo et $a l^{4}$ while allowing a much longer time for the vasculature to adapt. Myogenic responses of an autoregulated vessel may require several minutes to induce stable vessel diameters following an acute 
change in intravascular pressure, ${ }^{2021}$ while metabolic mechanisms seem to occur within a few seconds. ${ }^{23}$ Because the supine position was assumed for at least 30 minutes, there is good reason to be confident that control mechanisms had enough time to take effect in the present experimental set up and seem, in fact, to have influenced blood pressure in the ophthalmic artery. However, as in the study by Longo et al, because ChBF was measured with a device which assessed only the subfoveal choroid, the results presented hitherto may not apply to the remainder of the choroid.

In conclusion, our results show that subfoveal ChBF decreases proportionally with decreasing OPP, suggesting a passive response to changes in body posture. In contrast, the carotid system seems to regulate the perfusion pressure in its branches.

\section{Authors' affiliations}

P Kaeser, S Orgül, C Zawinka, G Reinhard, J Flammer, University Eye Clinic, Basel, Switzerland

\section{REFERENCES}

1 Kiel JW, Shepherd AP. Autoregulation of choroidal blood flow in the rabbit. Invest Ophthalmol Vis Sci 1992;33:2399-410.

2 Riva CE, Titze $P$, Hero $M$, et al. Effect of acute decreases of perfusion pressure on choroidal blood flow in humans. Invest Ophthalmol Vis Sci 1997;38:1752-60.

3 Hasler PW, Orgul S, Gugleta K, et al. Vascular dysregulation in the choroid of subjects with acral vasospasm. Arch Ophthalmol 2002;120:302-7.

4 Longo A, Geiser MH, Riva CE. Posture changes and subfoveal choroidal blood flow. Invest Ophthalmol Vis Sci 2004;45:546-51.

5 Riva CE, Cranstoun SD, Grunwald JE, et al. Choroidal blood flow in the foveal region of the human ocular fundus. Invest Ophthalmol Vis Sci 1994;35:4273-81.

6 Geiser MH, Diermann U, Riva CE. Compact laser Doppler choroidal flowmeter. J Biomed Opt 1999:4:459-64.

7 Riva CE, Cranstoun SD, Grunwald JE, et al. Choroidal blood flow in the foveal region on the human ocular fundus. Invest Ophthalmol Vis Sci 1994;35:4273-81.
8 Riva CE, Titze $P$, Hero $M$, et al. Choroidal blood flow during isometric exercises. Invest Ophthalmol Vis Sci 1997;38:2338-43.

9 Geiser MH, Riva CE, Dorner GT, et al. Response of choroidal blood flow in the foveal region to hyperoxia and hyperoxia-hypercapnia. Curr Eye Res 2000;21:669-76.

10 Kiss B, Dallinger S, Polak K, et al. Ocular hemodynamics during isometric exercise. Microvasc Res 2001;61:1-13.

11 Gherghel D, Orgul S, Dubler B, et al. Is vascular regulation in the central retinal artery altered in persons with vasospasm? Arch Ophthalmol 1999;117:1359-62.

12 Gugleta K, Orgul S, Flammer I, et al. Reliability of confocal choroidal laser Doppler flowmetry. Invest Ophthalmol Vis Sci 2002:43:723-8.

13 Preitner A Orgul S, Prunte C, et al. Measurement procedures in confocal choroidal laser Doppler flowmetry. Curr Eye Res 2004;28:233-40.

14 Sayegh FN, Weigelin E. Functional ophthalmodynamometry. Comparison between brachial and ophthalmic blood pressure in sitting and supine position. Angiology 1983;34:176-82.

15 Sayegh FN, Weigelin E. Functional ophthalmodynamometry. Comparison between dynamometry findings of healthy subjects in sitting and supine positions. Ophthalmologica 1983;187:196-201.

16 Pocock SJ, Assmann SE, Enos LE, et al. Subgroup analysis, covariate adjustment and baseline comparisons in clinical trial reporting: current practice and problems. Stat Med 2002;21:2917-30.

17 Orgul S, Meyer P, Cioffi GA. Physiology of blood flow regulation and mechanisms involved in optic nerve perfusion. J Glaucoma 1995:4:427-43.

18 Alm A, Bill A. Ocular and optic nerve blood flow at normal and increased intraocular pressure in monkeys (Macaca irus): a study with radioactively labelled microspheres including flow determination in brain and some other tissues. Exp Eye Res 1973;15:15-29.

19 Alm A, Bill A. The oxygen supply to the retina. II: Effects of high intraocular pressure and of increased arterial carbon dioxide on uveal and retinal blood flow in cats, a study with radioactively labelled microspheres, including flow determinations in brain and some other tissue. Acta Physiol Scand 1972:84:306-19.

20 Halpern W, Osol G. Influence of transmural pressure of myogenic responses of isolated cerebral arteries of the rat. Ann Biomed Eng 1985; 13:287-93

21 Rajagopalan S, Dube S, Canty JM. Regulation of coronary diameter by myogenic mechanisms in arterial microvessels greater than 100 microns in diameter. Am J Physiol 1995;268:H788-93.

22 Faraci FM, Heistad DD. Regulation of large cerebral arteries and cerebral microvascular pressure. Circ Res 1990;66:8-17.

23 Florence G, Seylaz J. Rapid autoregulation of cerebral blood flow: a laser-Doppler flowmetry study. J Cereb Blood Flow Metab 1992;12:674-80. 